\title{
Génesis semiótica de la intermedialidad: fundamentos cognitivos y socio-constructivistas de la comunicación
}

\author{
Asunción LóPEZ-VARELA AzCÁRATE*
}

Propuesto: 23 de mayo de 2011

Evaluado: 25 de mayo de 2011

Aceptado: 24 de mayo de 2011

(Abstracts y palabras clave al final del texto)

\section{INTRODUCCIÓN}

El proceso de transformación de la información de formatos analógicos a formatos digitales y la mayor facilidad de estos últimos para incorporar datos en distintos modos perceptivos (texto, imagen, audio, video) ha movilizado los estudios en torno al término "intermedialidad", es decir, el diálogo entre los antes distintos medios antes analógicos y que en la actualidad comparten un soporte digital común (para otros estudios sobre intermedialidad, véase Yalkut 1973, Higgins 1984, Frank 1987; Prümm 1988, Eicher 1994; Rajewsky 2002; Schröter 2008). El término podría haber sido utilizado por primera vez en 1983 por Hansen Löve, según indica Jens Schröter (2011), si bien "intermedia" aparecería ya en Samuel Taylor Coleridge. Según Dick Higgins, la separación entre los diversos medios se originaría en el Renacimiento (1984:18) como consecuencia de un deseo de purificación de los mismos. Por su parte, Peter Frank añade que es muy posible que la diversificación intermedial, además de a razones de índole tecnológica, se debiese a razones socio-políticas de especialización en las artes y las ciencias, coincidiendo con el establecimiento de cánones genéricos y academias diversificadas, competidoras entre sí.

Uno de los problemas fundamentales es la diferenciación que hace Higgins entre "intermedia" y "medios mixtos" (mixed media). Los segundos se podrían contemplar en su ontología como medios diferentes, mientras que los primeros asumen una fusión conceptual que no permite contemplarnos como separados en su origen (por ejemplo el cine). Sin embargo, tal y como indica Schröter (2008), tal síntesis solo tiene sentido entendida como creación-distribución-recepción que compartiría el mismo plano espacio-temporal, sujeta, por tanto, a implicaciones socio-culturales y de alguna manera institucionales. Es decir, que tal fusión tendría menos que ver con la síntesis intermedial, y sería más bien producto de asimilaciones cognitivas (lo que Schröter denomina Synthetic Intermediality). La importancia del contexto en el desarrollo del concepto de intermedialidad ya había sido puesta de relieve por Müller

* Universidad Complutense de Madrid. 
(1994:133), de forma que, para estos casos en los que se puede hablar de interpretaciones separadas de la forma material de los medios, Schröter emplea el término transmedial intermediality, afirmando que incluiría lo que Irina Rajewsky (2002) denomina "transposición". Así por ejemplo, Joaquim Paech afirma que "we could also say that there is no intermediality between literature and film; there is one only between media narrating literarily or cinematically" (Paech 1997: 335 traducción de Jens Schröter en su artículo de 2011). Esta afirmación, como menciona Arno Gimber, implicaría la transmedialidad de la narratividad como tertium comparationis (Gimber \& López-Varela), lo que permitiría abordar el estudio de la intermedialidad desde un punto de vista neo-formalista, tal y como ya había hecho Bordwell (1993) cuando indica que "subject patterning is independent of the medium; the same subject patterns could be embodied in a novel, a play, or a film" (50). Así, según indica Schröter, la homología estructural entre medios de distinto origen podría analizarse de forma transtextual, o mejor dicho, transmedial, lo que plantea problemas puesto que establece una noción jerárquico-dependiente entre la narratividad y la intermedialidad, dejando prácticamente de lado la especificidad del medio.

Existiría así una forma de concebir la intermedialidad, apreciable en el estudio historiográfico de la misma, y que supone el análisis del impacto dominante de ciertos medios en determinados momentos históricos. Un estudio de este tipo muestra como se han producido representaciones que han pasado de un medio en otro, lo que Bolter and Grusin (1999) han denominado "re-mediación", y para las que Schröter emplea el término transformational intermediality.

En resumen, la cuestión que se plantea es si estamos hablando de la existencia de materialidades específicas, dependientes de cada medio y que precederían a la relación intermedial o, si por el contrario, hablamos de una intermedialidad originaria (ontological intermediality en términos de Schröter) y que funcionaría como posibilidad de la existencia de unidades individuales (monomedia) y conjuntas (multimedia).

Mi investigación ha venido cuestionando aspectos similares a partir de una orientación investigadora en semiótica, literatura comparada y estudios culturales. Se trata de un tipo de exploración interdisciplinar que conlleva evidentes riesgos, puesto que uno no puede tener un conocimiento exhaustivo de todas las disciplinas. Sin embargo, en su conjunto, permite una visión comparativa que revela el impacto de los cambios tecnológicos en las formas de producción/representación, distribución, exhibición y recepción de la información, tanto en la evolución de las teorías científicas como en el curso de las investigaciones en ciencias sociales y humanidades (López-Varela 2004). Dichos cambios resultan particularmente evidentes cuando se atiende a las representaciones artísticas, mediáticas y científicas de vanguardia (López-Varela 2008).

Otro hecho que resulta cada vez más patente, tal y como menciona Gonzalo Abril (2010), es la eficacia de la movilización de los vínculos afectivos en los intercambios de información, lo que pasa por la mayor participación del lector, oyente o audiencia, tal y como señalarían ya las teorías de la recepción hace algunos años, llevando en la actualidad a una creciente intermedialidad, término que se emplea en las teorías de hipermedia y el análisis social de la información en Internet. Lo que Abril 
denomina "la exaltación de una (distante) proximidad", sería particularmente evidente en la nueva generación de aplicaciones de Internet, especialmente en las denominadas "redes sociales", pudiendo identificarse, tal y como hace Schröter (2010), con una democratización de los medios que llegaría incluso a afectar la conceptualización de fenómenos como la "intermedialidad", contemplándolos desde una perspectiva política.

Las publicaciones más recientes sobre el tema, ponen de manifiesto los condicionamientos socio-políticos del transporte de la información y su construcción conforme a imaginarios culturales, véase por ejemplo el estudio de Rodríguez-Virgili, Sádaba y López-Hermida (2010) en torno al impacto de la ficción en la comunicación política, o el libro de Alfredo Cramerotti (2009) sobre la movilización del concepto de verdad en el reportaje periodístico, desde el ámbito de la noticia, hacia el arte y la estética de la ficción. Este movimiento hacia lo cultural en el transporte de la información no es, sin embargo, tan fácilmente separable de los vehículos de la misma, es decir, del impacto de los cambios tecnológicos y la materialidad de los propios medios. Es este reconocimiento el que dirige mi orientación hacia la búsqueda de modelos que sustenten explicaciones materiales del propio desarrollo cognitivo-semiótico y cultural del ser humano.

\section{LA MATERIALIDAD DE LA CULTURA}

Todo fenómeno comunicativo responde a topologías, ecologías y tecnologías particulares que darían forma a nuestras ideas, ideologías, etc. El imaginario cultural contemporáneo parece situar el énfasis en el dialogismo, la intertextualidad, la intermedialidad, el hibridismo y la ambigüedad. Esta situación me ha hecho cuestionarme la validez científica de tales análisis y ha dirigido mi investigación hacia la exploración de aproximaciones más cercanas a los aspectos materiales de la comunicación humana con el fin de explorar hasta qué punto impactan sobre el imaginario cultural.

Mis trabajos anteriores se han centrado en explorar simultáneamente tendencias biológicas y culturales de los intercambios de información (véase López-Varela 2004). Más recientemente he intentado mostrar cómo la comunicación interpersonal es el soporte natural del desarrollo de la conciencia humana y como existe un amplio espectro de estudios en distintas áreas que muestran cómo la separación entre la mente individual y las mentes de los demás comienza a estrecharse ${ }^{1}$.

La mutualidad de experiencias compartidas se nos ofrece en cada encuentro diario y toma distintas formas materiales: desde una mirada o un gesto, a la utilización de signos más abstractos mediados a distancia de forma tecnológica. En diversos tra-

1 Ciencias cognitivas (Donald; Gallese; Trevarthen; Zlatev, Racine, Sinha \& Itkonen), psicología (Lacan, Laing, Stolorow, Atwood, Frie, Stern, Reis), lingüística (Wittgenstein, Benveniste, Levinas, Jacques, Fauconnier, Verhagen, Davidson), narratología (Bakhtin, Sternberg), educación (Vygotsky, Wertsch) sociología y antropología (Mead, Buber, Foucault, Schutz, Evans-Pritchard, Crook), filosofía (Dewey, Taylor, Habermas) semiótica (Peirce, Merleau-Ponty, Lotman), por nombrar solo algunas. 
bajos he estudiado el concepto de intersubjetividad como fundamento ontológico tanto para el desarrollo cognitivo intrapersonal como para la experiencia semiótica entre sujetos distintos y la interacción con nuestro entorno. El fenómeno intersubjetivo representa una experiencia del Otro que engloba aspectos perceptivos, emocionales, cognitivos, motivacionales y agentivos, y que surge a partir de emociones compartidas, atención conjunta, consciencia de la misma, además de intenciones congruentes; de ahí que los estudios en intersubjetividad sean lugar de encuentro de distintas áreas de investigación y de metodologías diversas. Hasta el momento me he aproximado al concepto desde una perspectiva psicológico-filosófica (LópezVarela 2010) y desde una valoración del impacto de las teorías de metáfora conceptual (López-Varela 2011). Otros trabajos han explorado los aspectos agentivos en la creación de marcos espacio-temporales cognitivos (López-Varela y Tötösy 2009; López-Varela y Net 2009), y más recientemente hacia el estudio de la intersubjetividad desde el ámbito de la neurociencia (López-Varela 2011).

Tales orientaciones no suponen la presunción de un esencialismo, durante años dominante en las ciencias dedicadas al estudio de lo que podríamos denominar "materialidad". Mis inquietudes parten de una visión compleja, muy en línea con el pensamiento de Edgar Morin. La visión compleja contempla los fenómenos vitales y humanos como procesos, abiertos al intercambio del información con el entorno que nos rodea, ya sean las realidades físicas (objetos y seres del mundo) o realidades psicológicas (conscientes o no conscientes). Tales intercambios son siempre mediados de manera semiótica a través de procesos cognitivo-emocionales distintos, a partir de medios exclusivamente biológicos o bien con ayuda tecnológica lo que permite encuentros a distancia. En mi opinión, el problema va más allá del estudio de cómo se produce el salto de la comunicación intrapersonal a la interacción interpersonal (y de ahí a la interculturalidad). Se trataría más bien de localizar el momento en que tiene lugar la diferenciación entre del ser social y el ser individual hasta el punto de que la comunicación con los Otros resulte problemática. Al mismo tiempo, habría que estudiar en qué medida los condicionamientos materiales tienen un impacto cultural, de manera que puedan haber llevado a propiciar tal separación. De la misma forma, el cuestionamiento podría trasladarse a la investigación tecno-cultural sobre paradigmas distintos (Kuhn), epistemes diferentes (Foucault) y aspectos de incompatibilidad lingüística (incommesurabilidad) e interdisciplinar (LópezVarela, en preparación).

\section{FUNDAMENTOS ONTOLÓGICOS Y EPISTEMOLÓGICOS DE LA INTERSUBJETIVIDAD Y SU RELACIÓN CON LA INTERMEDIALIDAD}

El estudio del desarrollo cognitivo humano a partir de metodologías filosóficas ha bifurcado a partir de dos metodologías aparentemente irreconciliables: por un lado la epistemología cartesiana de la primera persona (a partir del sujeto pensante), por otra las concepciones empiristas (Hobbes, Locke, Hume) que contemplarían la realidad a partir de la objetividad de la tercera persona (desde el objeto percibido). En su Crítica de la razón pura, Kant intentó demostrar cómo el sujeto cartesiano no 
es accesible a sí mismo puesto que su ocurrencia (sujeto de experiencia) tiene lugar en la mediación de sí-mismo como objeto de memoria, permaneciendo el "yo" como significante transcendental. Para superar el relativismo de la versión empirista, Christian de Quincey (2005) plantea que una forma empirismo intersubjetivo permitiría la introducción de una estructura tripartita — yo-tú-él/ella - en el proceso, a través de la cual sería posible el desarrollo cognitivo humano mediante la identificación con los Otros. A diferencia de las aproximaciones de Husserl (1970:185-6), el proceso de inter-relación no se inicia en la percepción fenomenológica intrasubjetiva del sujeto, sino que el "yo" tiene ya una consciencia agentiva como respuesta a las actitudes de Otros (Mead 1967: 175). Es decir que el "yo" se constituye como sujeto en su relación con los demás, a través de los valores, actitudes, creencias, sentimientos, aspiraciones y proyectos que surgen de manera socio-interactiva (Heidegger 2005: 149, 157, 162, 181; Sartre 1956: 51-155 y 160-161). Sin embargo, estas posturas todavía no ofrecen la explicación de una ontología no individualizada, es decir, una auténtica intersubjetividad, algo que Søren Overgaard (2007) ha continuado explorando en sus estudios de Sartre, Levinas, Wittgenstein, Heidegger y Husserl. La filosofía de Martin Buber pondría este acento en el aspecto dinámico de tales relaciones en torno a estructuras tripartitas vinculadas al desarrollo del lenguaje humano, tal y como formularían también Jacques Lacan y Charles Sanders Peirce desde distintos ámbitos. Estaríamos, por tanto, no solo ante una concepción dinámica de la ontología del yo, sino ante una revolución epistemológica en la que la comprensión deja de ser simplemente un modo sencillo de conocimiento para convertirse en una forma de ser, y donde los textos de los Otros se experimentarían como un momento de desposesión del yo narcisista, en lugar de como un momento de apropiación. (Ricoeur 1969).

Si bien, tal y como afirmase Maurice Merleau-Ponty el cuerpo es nuestro medio natural para construir el mundo ("le corps est notre moyen general d'avoir un monde") y los seres humanos tenemos la capacidad de crear extensiones artificiales comunicativas más allá de nuestras capacidades puramente biológicas. Posiblemente la diferencia fundamental con otros animales sea nuestra consciencia agentiva, cuya base puede vislumbrarse en la estructura argumentativa del lenguaje humano, tal y como ha señalado John Searle: "the intentionality that exists in each individual head has the form "we intend"'. (Searle 1995: 26) A partir de ese momento, es fundamental contemplar la comunicación como un proceso de intercambio de información que requiere de interacciones intencionales entre diversos materiales de transducción que pueden variar en su naturaleza, y que son tanto biológicos (el cuerpo humano) como tecnológicos.

\section{NEURONAS ESPEJO E INTERSUBJETIVIDAD}

Uno de los aspectos fundamentales de las investigaciones sobre intersubjetividad tiene por objeto explorar cómo y cuándo tiene lugar la diferenciación subjetiva. Es decir, la comunicación intersubjetiva va más allá de las meras respuestas empáticas o validación consciente mediante el intercambio de señales entre sujetos dis- 
tintos e independientes. Estamos hablando de invertir el orden epistemológico y partir de procesos dinámicos interactivos desde los cuales tendría lugar la diferenciación. La intersubjetividad sería, por tanto, una propiedad de todo sujeto que se constituiría en su interacción con los demás, una interacción orgánica y mutualmente comunicativa, co-creativa y co-dependiente.

El problema de toda visión procesal y dinámica compleja es, y ha sido siempre, establecer su ontología. La pregunta clave es, por tanto ¿dónde y en qué momento surge la matriz de interrelaciones? Investigaciones neurocientíficas recientes (Zlatev, Racine, Sinha, and Itkonen 2008; Morganti, Carassa, y Riva 2008) muestran cómo el desarrollo cognitivo del cerebro humano tiene lugar a partir de neuronas espejo que reflejarían la información perceptiva de unos modos a otros, fundamentalmente a través de relaciones motoras e intencionales. Ello es posible por el organismo adquiere información de manera simultánea a través de distintos modos perceptivos. Tales estructuras neuronales se encontraron en la última década del siglo XX en primates a través de los estudios desarrollados en la Universidad de Parma (Italia) por Giacomo Rizzolatti, Leonardo Fogassi y Vittorio Gallese (2009). Posteriormente, técnicas como la simulación magnética transcraneal y la electroencefalografía han permitido encontrar evidencias de sistemas similares en el cerebro humano.

En una primera fase, el contacto afectivo entre madre y bebé es fundamental para el desarrollo exteroceptivo (a través de los sentidos con los que percibimos el mundo exterior - visión, gusto, olfato, tacto, audición y equilibriocepción). Durante una fase de imitación, fundamentalmente mediada por la visión, las neuronas espejo copiarían información de los movimientos de los Otros (madre, padre, familia cercana). Poco a poco se produciría una fase agentiva de imitación en la que posiblemente el bebé no sea de todo consciente de sus actos.

Tras el primer contacto visual y las primeras sonrisas, vendría una fase de desarrollo propioceptivo, sentido en el que se tiene conciencia del estado interno del cuerpo a través, de neuronas sensoriales del oído interno (movimiento y orientación) y receptores de estiramiento de los músculos (postura). La propiocepción interviene en el desarrollo de lo que se ha denominado "esquema corporal" (body schema) (Gallagher 2009). Los primeros movimientos de la cabeza a través del sistema vesticular proporcionan orientaciones espaciales. El sistema cutáneo comienza a registrar información relacionada con el tacto (temperatura, dolor, placer...) permitiendo la discriminación de estímulos y participando en el desarrollo del conocimiento del cuerpo, los vínculos emocionales, y el sentido de seguridad personal. Si se producen disfunciones sensoriales en este sistema, se pueden originar dificultades para interaccionar con el medio, torpeza motriz tanto en los desplazamientos como en las manipulaciones de materiales y herramientas, desorganización e incluso retraso en la adquisición de independencia personal. Las consecuencias a nivel afectivo-emocional pueden ser una falta de organización temporal y secuencial (ritmos vitales alterados), hiperactividad, inestabilidad y dificultad en las relaciones sociales.

Los gestos y movimientos de las manos agitando y dejando caer objetos desarrollan poco a poco funciones de acción motora planificadas a través de sensaciones de kinestesia que se transmiten desde todos los puntos del cuerpo a los centros 
nerviosos, regulando el equilibrio y las sinergias (acciones voluntarias coordinadas) necesarias para llevar a cabo cualquier desplazamiento del cuerpo. Los primeros pasos contribuyen a regular mejor este control del equilibrio y la coordinación de ambos lados del cuerpo.

Toda la información que recibe el organismo en movimiento resulta en representaciones multimodales (varios modos perceptivos) de las acciones. Además la comprensión de toda acción sigue patrones multi-tarea, puesto que por una parte la percepción registra los comportamientos de los Otros para con el mundo que les rodea (por ejemplo les ve realizar acciones que luego se pueden copiar) y registra también los aspectos menos obvios, como las razones y motivaciones que obligan a una persona a actuar de una manera determinada. Es decir que el ser humano es capaz de comprender la acción intencional de los otros al percibir sus actos de tal manera que, a diferencia de otros animales, los seres humanos son criaturas conscientemente agentivas (Zlatev, Racine, Sinha, and Itkonen 2008: 222)

Jordan Zlatev proporciona una explicación para el desarrollo cognitivo humano vinculado al desarrollo emocional (intersubjetividad) y distingue cinco niveles de lo que denomina "mímesis corporal". Los primeros intercambios comunicativos son copias de representaciones mentales, aunque no son intencionales. Se trata por ejemplo de las miradas y las sonrisas de los recién nacidos. Poco a poco se pasa a una fase de imitación en la que el bebé comparte la atención de las miradas y es capaz de reconocerse en el espejo como ser distinto con su propio "esquema corporal" (body schema) que, de alguna forma, es innato en el sentido de que está ya presente en el momento del nacimiento y es pre-consciente. Desde el punto de vista neurológico, durante ambas fases se producen cruces multimodales en la forma en que las percepciones se almacenan en el cerebro, conectando lo que se denomina exterocepción y la interocepción/propiocepción. Las neuronas-espejo se comunican a través de sustancias químicas que se segregan de manera equivalente para relaciones intencionales distintas, lo que hace posible, desde el punto de vista fisiológico (y no solo cultural), la existencia de representaciones compartidas por personas distintas.

A diferencia del body schema la imagen corporal body image se desarrolla con la diferenciación entre sujeto y objeto y es, hasta cierto punto, consciente. El sistema somatosensorial se activa cuando las neuronas sensoriales son impulsadas por algún estímulo específico, como el calor; las neuronas pasan a un área del cerebro especialmente atribuida al área del cuerpo que ha recibido el estímulo. El mapeo de la superficie corporal en el cerebro se denomina homúnculo y es esencial en la creación de la imagen corporal. En estas fases se desarrolla la acción intencional, antes incluso que los primeros movimientos corporales (agitar, dejar caer objetos). El desarrollo de la atención requiere la habilidad para cambiar de perspectiva entre distintos ejes, atender al foco de atención, a cómo los demás atienden al mismo, y a cómo son también conscientes de la atención de los demás.

La fase que Zlatev denomina "triadic mimesis" consiste en la utilización de signos más allá de gestos, miradas y sonidos de placer y dolor (iconos e índices). Comienzan a utilizarse signos consensuados complejos cada vez más abstractos: dibujos y lenguaje común, que muestran aspectos de cooperación/co-creación. Los 


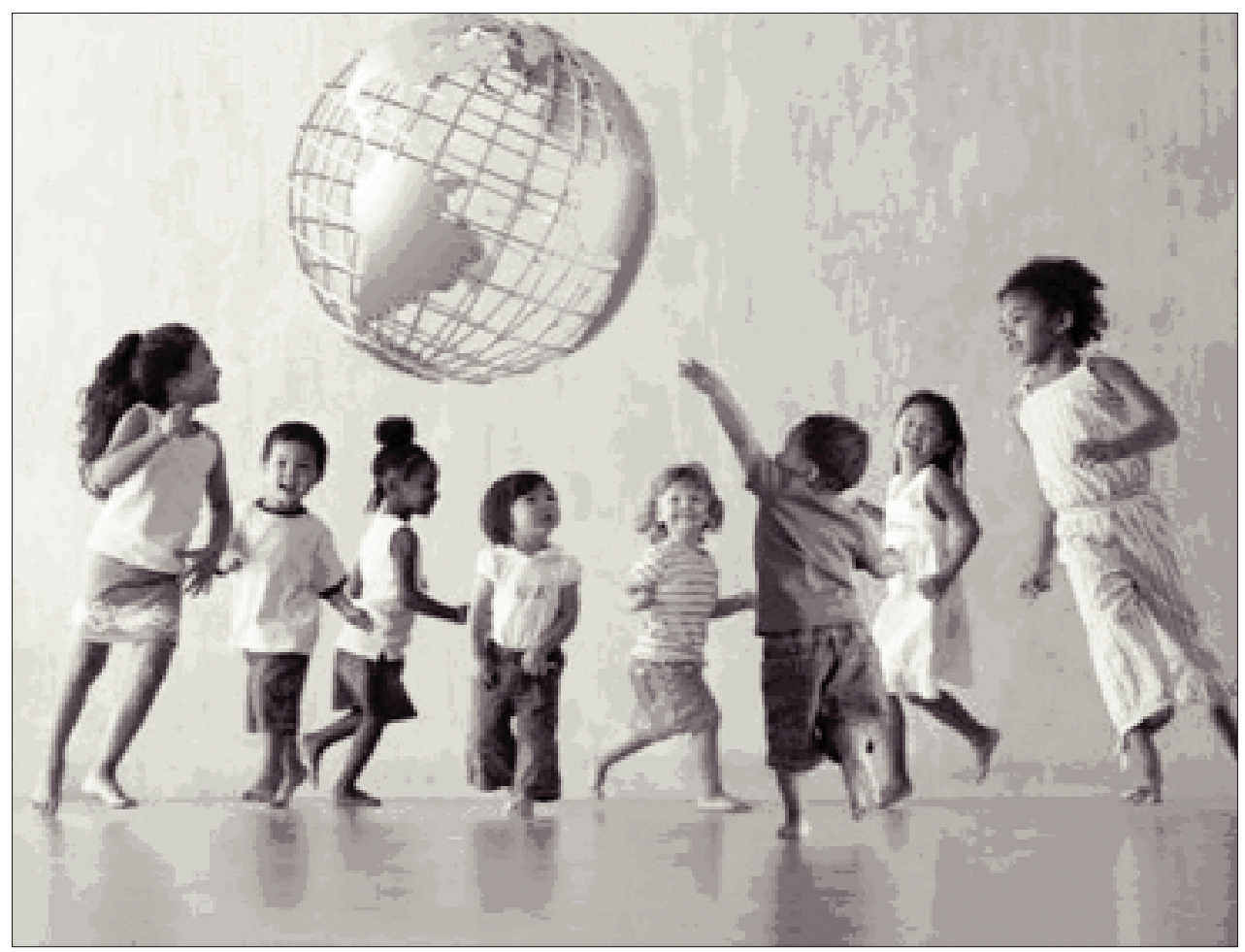

estudios de Shaun Gallagher (2009), Daniel Hutto (2009), John Barressi y Chris Moore (2011), entre otros, muestran la importancia del desarrollo de la narratividad como estructura cognitiva. Aparentemente la repetición de estructuras narratológicas (a través de cuentos e historias en la infancia) contribuye también al desarrollo mental a través de estructuras de imitación, que a nivel neurológico se estructuran como interacciones ficticias entre sujeto y objeto, y que corresponderían a acciones intencionales reales. Con el desarrollo de la capacidad lectora aumentaría las posibilidades de interacción neuronal multimodal, lo que en términos coloquiales se denominaría también "imaginación" o capacidad de preveer intenciones-acciones futuras.

\section{LENGUAJE, CONSTRUCCIONES EMOCIONALES Y NARRATIVIDAD}

Donald Davidson ha señalado la importancia de la adquisición del lenguaje en el fenómeno denominado "triangulación" semiótica, "that involves two or more creatures simultaneously in interaction with each other and with the world they share," and where "each correlates their own reactions to external phenomena with the reactions of the other." (2001: 128-9) El lenguaje sería esencial para el desarrollo cognitivo de orden superior porque "unless the base line of the triangle, the line betwe- 
en the two agents, is strengthened to the point where it can implement the communication of propositional contents, there is no way the agents can make use of the triangular situation to form judgments about the world; only when language is in place can creatures appreciate the concept of objective truth" (2001: 130). Es decir que, como ya afirmaba Wittgenstein, la habilidad para comunicarse con los demás a través del lenguaje subyace en la capacidad de desarrollar pensamientos sobre el mundo. De ahí que la posibilidad de describir el mundo de una manera objetiva (consensuada) dependa de nuestra capacidad de comunicación intersubjetiva.

El primer "registro" de lo psíquico es, de acuerdo con Lacan, la dimensión nolingüística o "lo imaginario", fundamentado en una forma de cognición a través de imágenes, iconos en el sentido semiológico. La exterocepción deja huellas o marcas psíquicas (signos perceptivos) que conforman un espacio psíquico compuesto de imágenes provenientes primero de todos los sentidos y, posteriormente de los movimientos del otro y del propio cuerpo (propiocepción). Cuando logran significarse como propios, producen una imagen integrada del sujeto (body image) que pasa a comprenderse como uno, distinto de otro. Tal y como hemos visto, tales estructuras mentales son en su principio, fundamentalmente imitativas, teniendo lugar de manera fisiológica a través del estructuras topológicas mentales formadas por neuronas espejo. Sin embargo, en determinado momento, y como consecuencia del nacimiento de la consciencia del yo, se desarrollan como estructuras propias y diferenciadas. A partir del denominado estadio del espejo, en torno a los seis meses de edad según Lacan, el sujeto es capaz de identificar su imagen como un "yo" distinto. El niño contempla su imagen especular como un todo, si bien la falta de coordinación motora de su cuerpo le lleva a percibirse como un "yo" diferenciado pero incompleto. Este contraste entre la imagen percibida y la experiencia sensorial crea una tensión entre el sujeto y la imagen que percibe de si mismo. Para resolver esta tensión, el sujeto se identifica con la imagen dando lugar a la formación de lo que en psicología se denomina "ego". En su relación con el objeto más importante percibido, su madre y la omnipotencia de la misma, el niño se siente un ser alienado de sí mismo, creándose la imagen que tiene de sí mismo en el orden Imaginario, como posibilidad hipotética. En sus primeros trabajos, Lacan concibió la intersubjetividad como transindividual. Posteriormente comenzó a asociarla con nociones de reciprocidad y simetría, es decir, con lo imaginario (Lacan 1991: 20).

El desarrollo motor, que Lacan describe con un girar la cabeza hacia un tercero, el padre (Seminario III, 1956 y X, 1962-63), iría emparejado con el nacimiento del orden Simbólico, que se origina por mediación del lenguaje y a instancias de la aparición de estructuras triangulares afectivas, lo que denomina "el nombre del padre". Durante esta etapa y antes de los cuatro años, el padre será inscrito como significante, con ayuda de la mediación de la madre y a través del lenguaje. El padre se convierte en el artífice de la ley u orden simbólico (logos). Si bien el registro de lo simbólico es probablemente una capacidad innata en el sentido de encontrarse presente en el momento del nacimiento, Lacan deja claro que su despliegue sólo es posible a partir de un estímulo específico, la función paterna. En los casos de psicosis, que Lacan investigaba cuando formuló su teoría de lo simbólico, se rechazaría inconscientemente la inscripción del padre como signo, de manera que se produce una exclusión de un sig- 
nificante fundamental, proceso mucho más radical que la represión ("forclusión" es el término que Lacan emplea y a partir del cual define lo real como un lugar otro de lo psíquico donde los significantes forcluidos reaparecen en forma de alucinaciones).

Las formulaciones de Lacan sobre el estadio del espejo, aunque incompletas, pueden considerarse geniales aproximaciones, precursoras de los estudios de psicología cognitiva y evolutiva. Como hemos visto, muchas de sus ideas muestran claros paralelismos con descubrimientos neurocientíficos más recientes. Dentro de los estudios de semiótica, son interesantes las relecturas de Lacan que ha realizado Julia Kristeva con el fin de incluir a la madre de manera más clara en todo el proceso de desarrollo cognitivo. Sería interesante ampliar el estudio del lenguaje femenino, caracterizado según Kristeva por una mayor ambigüedad semiótica, y ver hasta qué punto la formación de significados emocionales, más connotativos que denotativos, y muy vinculados a la musicalidad y prosodia del lenguaje, ocupan una posición primaria frente al orden simbólico paterno. Tal estudio queda fuera del ámbito de este artículo, si bien las líneas siguientes ofrecen una guía de la orientación futura de mi investigación.

Quedarían, pues, dos aspectos fundamentales por abordar, que solo puedo esbozar aquí. Por una parte se encuentra el estudio de las estructuras de memoria, que según el tipo de modalidad perceptiva, sigue patrones fragmentados o, por el contrario, teleológicos. Por otra, y en cierta forma relacionada con la anterior, se sitúa la investigación de los aspectos emocionales circunscritos a la comunicación. Como he intentado mostrar de manera sucinta, el desarrollo psicológico-cognitivo depende en gran parte de las relaciones afectivas que se crean en el entorno familiar. La propia etimología de la palabra e-moción, pone de manifiesto la estructura dinámica del concepto, ya contemplado por Platón, Aristóteles, Descartes o Spinoza entre otros filósofos (véase Knuuttila 2004) vinculado a la movilización del pensamiento racional. Incluso antes de los estudios de Freud sobre la acción emocional y su capacidad de plasmar estructuras psíquicas profundas en el inconsciente (que se vislumbran con frecuencia a través de la creatividad y el arte), Charles Darwin vinculó el estudio de los afectos a su teoría de la evolución, publicando un libro sobre la Expresión de las emociones en los humanos y los animales.

Estudios más recientes sobre la naturaleza de las emociones (por ejemplo Oatley and Jenkins 1996) muestran que son fundamentales en la socialización, cumpliendo una importante acción comunicativa interpersonal, puesto que se estructuran siempre en relación a los Otros. Las emociones suponen valoraciones de ocurrencias pasadas a través de la acción de la memoria selectiva (el recuerdo), que es un tipo de memoria que registra encadenamiento de hechos bajo un patrón dinámico (causaefecto), es decir, de forma narratológica, con el fin de establecer prioridades en la acción futura. Las emociones ordenan los hechos que se consideran de mayor importancia tanto para el individuo como para su comunidad, determinando la urgencia de las preocupaciones e intereses, y por tanto, la forma en que las personas interactuamos con el mundo.

Las dos cuestiones planteadas más arriba pueden abordarse de manera conjunta, si bien como adelantábamos, este artículo presenta únicamente un esbozo. Por ejemplo, en narratología, la importancia del estudio de las emociones queda de manifiesto en los trabajos de Meir Sternberg, que viene desarrollando diversas formulacio- 
nes para explicar lo que denomina protean fact/feeling inter-dynamics (Sternberg 2003: 360) en la retórica del hecho narrativo. De manera indirecta, tal estudio, que abordamos en un volumen monográfico en preparación, supone una revisión de la ontología del criterio de "verdad", y de las distinciones entre ficción/no-ficción (narrativa histórica, autobiografía, reportaje, etc.) en torno a la acción del sujeto que recuerda, narra y emplea marcadores deícticos que localizan las acciones en contextos espacio-temporales determinados.

Esta investigación en narratología discurre en paralelo a los descubrimientos en neurociencia, donde Ronald Rensink o Zenon Pylyshyn, por ejemplo, han mostrado cómo uno de los mecanismos más básicos para la selección de información visual es el procesamiento de estímulos dentro de un espacio limitado mediante la asignación de índices o demostrativos. En una primera fase (working memory phase), contemplamos los objetos que nos rodean como proto-objetos con los que interactuar en un futuro (task-oriented capacity); de manera que pasan a ser objetos mentales (sin necesidad de ser clasificados como signos. Esta memoria de trabajo hace solo un uso dinámico de las variables deícticas, futuros objetivos de actividad motora. De acuerdo con Pylyshyn, "the pointer has to point to an object, rather than a location, in order for this to function in dynamic scenes. Such a pointer would allow the system to map a newly perceived property onto a representation of the object that had been previously (incompletely) encoded". (2000: 200) En otras palabras, los objetos son detectados en el imaginario, sin llegar a ser conceptualizados en el nivel simbólico, donde se produce la codificación de las propiedades sensoriales. Se trata, pues, de una estrategia inconsciente, evidente también en los mecanismos de lectura superficial, que permite obtener un cierto grado de información con un vistazo rápido, algo fundamental en un entorno cambiante como el que nos rodea.

Otros estudios cognitivos muestran la relación en las operaciones de visión y tacto y la contigüidad espacial. Así, por ejemplo, la presentación intermedial de texto e imagen en un mismo documento funciona mejor cuando se encuentran cercanos. En el caso de los cómics y de las novelas gráficas (para un resumen los mecanismos de metáfora conceptual y una aplicación a la novela gráfica se puede consultar López-Varela 2011b), la colocación del texto dentro de la viñeta es la opción que más operativa desde el punto de vista de procesamiento mental, aunque si se coloca bajo ella es suficiente. Sin embargo, el texto colocado fuera de la viñeta no se procesa con facilidad de manera conjunta. En el caso del sonido, características como la intensidad, el tiempo, la articulación y el timbre, cumplen también funciones primariamente deícticas, vinculadas a los ritmos biológicos (López-Varela en preparación para Sonic Futures $<$ http://www.necs-initiative.org/>)

El segundo aspecto de la investigación, tal y como mencionábamos líneas más arriba, se refiere a los avances en el estudio del impacto de las estructuras narrativas y el lenguaje en relación con la intersubjetividad y los aspectos emocionales. Así, por ejemplo, los trabajos de John Barresi y Chris Moore (2008) con personas autistas, muestran la incapacidad de las mismas para establecer relaciones cognitivas que incorporen acciones comunicativas triangulares, es decir, que muestren la relación mental entre el punto de vista del sujeto (primera persona) y del objeto (tercera persona). Conceptos abstractos, aunque públicos, como el amor pueden interpretarse al 
percibir cierto tipo de comportamientos que el sujeto dirige hacia el objeto de su cariño, y que éste devuelve de alguna manera. En el caso del autismo, problemas en el desarrollo de neuronas espejo traerían como consecuencia la incapacidad de integrar información entre sujeto y objeto, entre "yo" y los Otros. Otros autores, como Daniel Hutto (2008) y Shaun Gallagher (2009), han hecho notar que la práctica narrativa en la infancia tendría un papel fundamental en el desarrollo de la capacidad intersubjetiva, y por tanto, en el desarrollo cognitivo posterior.

Desde el ámbito sociológico, estudios como los de James Gross (2006) muestran como la comunicación emocional es un importante indicador de la cooperación entre los miembros de un grupo. Una relación cercana propicia la credibilidad y la confianza. En los contextos mediados (no presentes de manera inmediatamente física), la credibilidad depende de la labor enunciativa del sujeto (lo que en narratología se denomina "punto de vista narrativo"). Durante años, los aspectos emocionales se habían relegado a los ámbitos del arte y de la ficción, intentando acercar la no-ficción a un criterio de verdad objetiva que solo existe como probabilidad. En la actualidad, las investigaciones sobre inteligencia emocional han recobrado impulso. En 1993 Howard Gardner comenzó a hablar de inteligencias múltiples, entre las que destacaba la inteligencia interpersonal. Dos años después Daniel Goleman hablaba de inteligencia emocional. En el mundo contemporáneo, con multinacionales que trabajan cada vez más de manera transnacional y translocalizada, y por tanto transcultural, muchos estudios muestran la relevancia del desarrollo de estructuras intersubjetivas en el ámbito empresarial y contextos organizativos, particularmente en posiciones de liderazgo (Higgs and Hender 2004). Se señala la importancia de desarrollar la capacidad de examinar las percepciones de los otros y de empatizar con sus emociones con el fin de potenciar las posibilidades de éxito empresarial (Weisinger 1998: 214-5).

En el ámbito educativo, el trabajo de James V. Wertsch, desarrollado a partir de las nociones de "pensamiento en acción" del psicólogo ruso fundador del socioconstructivismo Lev Vygotsky, considera la acción mediada como una relación contextual (situada históricamente en un espacio-tiempo determinado) entre agentes humanos y sus artefactos culturales (tecnologías) (para una revisión de estudios publicados en este contexto véase López-Varela 2008 y López-Varela y Tötösy 2009). La correlación (mediada) entre signo y artefacto, entre concepto y procedimiento, hace que, cuando los artefactos cambian, también lo hagan los signos o representaciones culturales. Para Wertsch la comunicación emocional se aprende no solo en el ámbito familiar sino también en el educativo e institucional. El desarrollo emocional se produce vinculado a determinada cultura, dentro de la cual el individuo muestra su solidaridad para con una cierta comunidad de creencias, valores, etc., y utiliza determinadas tecnologías significativas.

\section{RELACIONES INTERMEDIALES}

Un aspecto fundamental de la investigación en intermedialidad es el concepto de medio. Desde que Marshall McLuhan (1962) contemplase los medios tecnológicos 
como extensiones del cuerpo, el debate en torno a esta cuestión ha estado servido. Mientras hay quien considera que "communication is a pipeline transfer of units of material called 'information' from one place to another" (Ong 1982:176), la transformación de los formatos analógicos en digitales ha venido a complicar todavía más la discusión. Así, por ejemplo, aunque Lev Manovich emplea el término "new media" (2001: 52), Janet Murray cree que se presta a interpretaciones: "[T]he term 'new media' is a sign of our current confusion... How long will it take before we see the gift for what it is - a single new medium of representation, the digital medium, formed by the braided interplay of technical invention and cultural expression at the end of the 20th century?" (Murray 2003:3) En relación con el comentario de que "el contenido de un medio es siempre otro medio" (McLuhan 1962: 23) Jay David Bolter y Richard Grusin denominan "the representation of one medium in another remediation, and we will argue that remediation is a defining characteristic of the new digital media. What might seem at first to be an esoteric practice is so widespread that we can identify a spectrum of different ways in which digital media remediate their predecessors, a spectrum depending on the degree of perceived competition or rivalry between the new media and the old." (1999: 45) y vid Bolter (2011), De esta forma, la 'medialidad' se extiende metonímicamente para designar "the interaction of technology, society, and cultural factors through which institutionalized media of communication [...] produce, transform and circulate symbols in everyday life" (Friesen and Hug 2009: 69).

El factor común en todas estas definiciones es la integración de uno o varios medios en alguna otra forma de comunicación. Esto indicaría que estamos hablando de construcciones complejas que implican no solo distintas formas físicas de codificar la experiencia humana, sino de una amalgama de relaciones culturales (procesos) que incluyen diversos canales de procesamiento de la información obtenida a partir de esas experiencias (visuales, auditivas, etc.), y que se mueven también en torno a cambios en los contextos comunicativos (códigos distintos, relaciones diversas entre productores y receptores de la información, canales de distribución variables...) (Mayer 2005: 54).

Junto con la palabra "medio", el término "intermedialidad" ha generado su propio debate. El prefijo latino "inter" hace referencia a algo que se comparte entre varias opciones pero, al mismo tiempo, indica mutualidad y reciprocidad. Las primeras referencias al término aparecen en la Poética de Aristóteles, pero se atribuye a Dick Higgins su utilización más o menos novel en el ensayo de "Intermedia" (1966). Como mencionábamos al comienzo de este trabajo, y según apunta Irina Rawjesky, habría dos aproximaciones distintas al estudio del la intermedialidad. Por un lado, una orientación derivada de los estudios literarios y narrativos, en paralelo con el desarrollo de conceptos como dialogismo e intertextualidad, presentes en la obra de Michael Bakhtin y en las relecturas de la misma a partir de las traducciones de Julia Kristeva y los trabajos críticos del grupo Tel Quel (véase por ejemplo Pfister; Plett, Wolf; Balme, Müller). Por otro, se encuentra la orientación que deriva de los estudios de comunicación y que se centra en las distinciones materiales entre los medios y los grupos de fenómenos asociados a ellos (Spielmann; Schröter; Gaudraultt y Marion; Paech, Chapple y Kattenbelt). Rajewsky distingue entre rela- 
ción intramedial (y por lo tanto intertextual) e intermedial. Dentro de las primeras, un medio evoca y genera una ilusión de prácticas específicas de otro medio (por ejemplo el capítulo "Sirenas" del Ulises de James Joyce intenta evocar una composición musical denominada fuga). Según sus posibilidades de combinación, hay medios que solo pueden ser transpuestos o recreados unos en los otros (monomediales), mientras que hay otros que son plurimediales, como la danza o el teatro. Para Lars Elleström (2010) la cuestión no es solo la diferencia en los 'medios técnicos', sino que se trata de entender la intermedialidad como un punto de encuentro entre los aspectos materiales, semióticos, culturales, e incluyendo la variabilidad espaciotemporal (tanto histórica como material, por ejemplo el hecho de que la fotografía de paso al cine o 'motion pictures').

La crítica en hipermedia realizada por teóricos como George Landow y Katherine Hayles pone de manifiesto aspectos importantes en lo que se refiere a la conversión de los formatos analógicos en digitales. Las nuevas estructuras presentan características reticulares y, con frecuencia, reflexivas, de forma que, aparentemente, la lógica narrativa no serviría para explicar algunas de las relaciones que tienen lugar en muchas de las obras denominadas "literatura electrónica". Uno de los aspectos principales que forma parte de mi investigación-en-proceso es intentar mostrar de manera más clara la vinculación entre la noción de 'interactividad' y los procesos cognitivos con ella relacionados. En el caso de las operaciones en Red, no estamos hablando únicamente de percepciones intermediales que dan lugar a interpretaciones críticas de tipo asociativo-narratológico. Los medios digitales ofrecen una verdadera posibilidad de modificar la información y, por tanto, la acción comunicativa. En un nivel básico este tipo de interacciones es operable a través del teclado y del ratón (tacto), los altavoces y el micrófono (audio), la pantalla y la cámara Web (visión). Sin embargo, las tecnologías avanzan incansablemente hacia la realidad aumentada, la simulación y la realidad virtual.

\section{CONCLUSION}

Este ensayo ha buscado acercarse a los conceptos de 'medialidad' e 'intermedialidad' desde una perspectiva híbrida que comprende los estudios culturales, inquietudes cognitivas y semióticas, y una breve aproximación a los entornos digitales. De esta forma, a partir de la noción de intersubjetividad como fundamento cognitivo (en sus aspectos más materiales, es decir en sus formas biológicas, que incluyen aspectos fisiológicos y psicológicos), se ha pasado a relacionar la intermedialidad con el desarrollo tecnológico, modelado a partir de estructuras tomadas de los propios procesos biológicos, aunque sin perder de vista la acción cultural. Las extensiones comunicativas, en el sentido de Mc Luhan, deben contemplarse desde el punto de vista de la función interactiva humana, es decir, como artefactos funcionales, cuya acción comunicativa tiene lugar en un marco de intercambio sujeto-objeto, en torno a procesos sistémicos complejos que incorporan agentividad e intencionalidad tanto individual como grupal, de manera que dan "cuerpo" a repertorios de significaciones y prácticas culturales a través de lo que el sociólogo 
francés Pierre Bourdieu ha denominado habitus (1981: 134), y que mueven la disposición a interactuar de una manera determinada. El habitus es la generación de prácticas que están limitadas por las condiciones sociales que las soportan, es la forma en que las estructuras sociales se graban en el cuerpo y la mente humana y forman estructuras subjetivas sociales. Tal y como señalaría Mikhail Bakhtin en su aproximación filosófico-literaria al estudio del lenguaje: "Prior to the moment of appropriation the word does not exist in a neutral and impersonal language [...] but rather it exists in other people's mouths, in other people's contexts, serving other people's intentions: it is from there that one must take the word and make it one's own." (Bakhtin 1981: 294)

La novedad del enfoque aquí presentado consiste en la confrontación de elementos tradicionalmente considerados 'esenciales' y 'universales' y por tanto 'inmutables' (los modos biológicos de percepción y transmisión de la información), con patrones en continuo cambio, como son las manifestaciones culturales y los formatos tecnológicos. Se propone así una revisión de la ontología de la percepción y del potencial comunicativo humano, relacionando la investigación neurocientífica, los factores emocionales, los estudios culturales socio-constructivistas y, por último, la naturaleza cambiante de los medios técnicos, imprescindibles para toda comunicación a distancia.

En el contexto contemporáneo, inmerso en una movilidad continua tanto física como ideológica mediada por la velocidad de los medios de transporte de personas y de datos (Internet) a través no solo de las fronteras físicas de nuestro cuerpo sino también de las fronteras territoriales de nuestras comunidades, el impacto de la investigación en intersubjetividad (e interculturalidad) se encuentra necesariamente vinculado a los cambios en los medios materiales que propician los intercambios comunicativos, es decir, a la noción de intermedialidad. Confiamos en haber desvelado alguno de los hilos que forman parte del complejo entramado que vincula ambos conceptos, y en haber contado con la credibilidad del lector, a pesar de haberle llevado por caminos intrincados, siempre bajo la pauta de un hipotético "sentido común".

\section{REFERENCIAS BIBLIOGRÁFICAS}

ABRIL, Gonzalo (2010): "Cultural visual y espacio público-político", Cuadernos de Información y Comunicación. 15, pp. 21-36.

AlbersmeIER, Franz Josef and RolofF, Volker (eds.) (1989): Literaturverfilmungen. Frankfurt am Main: Suhrkamp.

AlbersmeIER Franz Josef (1992): Theater, Film und Literatur in Frankreich. Medienwechsel und Intermedialität. Darmstadt: Wissenschaftliche Buchgesellschaft.

BaKhtin, Mikhail (1981): The dialogic imagination: Four essays. Trans. M. Holquist. Austin, TX: University of Texas Press.

Balme, Christopher B. (2001): "Robert Lepage und die Zukunft des Theaters im Medienzeitalter." In Leeker, Martina (ed.) Machinen, Medien, Performances: Theater an der Schnittstelle zu digitalen Welten. Berlin: Alexander Verlag, Págs. 668-683. 
BArresi, John y MoOre, Chris. (2011) Understanding Intentional Relations of Self and Other. Oxford University Press.

Bolter, Jay David and Grusin, Richard (1999): Remediation. Understanding New Media. Cambdridge/MA: MIT Press.

Bordwell, David (1993): Narration in the Fiction Film. London: Routledge.

Bourdieu, Pierrre (1981): Questions de sociologie. Paris : Minuit.

Buber, Martin.(1970 / 1923): I and thou. Trans. R. Smith. New York: Charles Scribners Sons. (1965): The knowledge of man: A philosophy of the interhuman. New York: Harper and Row.

Chapple, Freda \& Kattenbelt, Chiel (eds.) (2006): Intermediatlity in Theatre and Performance. Amsterdam, New York: Rodopi.

CLÜVER, Claus (2001): "Inter textus / Inter artes / Intermedia," in Komparatistik. Jahrbuch der Deutschen Gesellschaft für Allgemeine und Vergleichende Literaturwissenschaft (2000/2001), Heidelberg, Synchron. Print.

Cramerotti, Alfredo (2009): Aesthetic Journalism. The University of Chicago Press.

DAvidson, Donald (2001): Subjective, Intersubjective, Objective. Oxford: Clarendon Press.

DE QuinceY, Christian (2005): Radical Knowing: Understanding Consciousness through Relationship. Park Street Press.

EICHER, Thomas (1994): "Was heißt (hier) Intermedialität?”. En Intermedialität. Vom Bild zum Text.: Thomas Eicher \& Ulf Bleeckman. (ed.) Bielefeld: Aisthesis, págs. 11-28.

Elleström, Lars (Ed.) (2010): Media Borders: Multimodality and Intermediality. Basingstoke, UK: Palgrave-Macmillan Ltd.

ERnst, Marc; Banks, Martin and Bülthoff, Heinrich (2000): Touch can change visual slant perception. Nature Neuroscience 3. 1 págs. 69-73.

FRANK, Peter (1987): Intermedia. Die Verschmelzung der Künste. Bern: Benteli.

Friesen, Norm and Hug, Theo (2009): "The Mediatic Turn: Exploring Concepts for Media Pedagogy". Ed. Friesen Norm and Theo Hug. Mediatization. . New York: Peter Lang. págs. 63-84.

Gallagher, Shaun (2009): "Two Problems of Intersubjectivity." Journal of Consciousness Studies $16, \mathrm{n}^{\circ} .6-8$, págs. 289-308.

GALlese, Vittorio (2009): "Mirror Neurons, embodied simulation and the neural basis of social identification.” Psychoanalytic Dialogues 19, págs. 519-536.

$<$ http://www.unipr.it/arpa/mirror/pubs/pdffiles/Gallese/Gallese\%20PD\%202009a.pdf>

GARDNER, Howard (1993): Multiple Intelligences: The Theory in Practice. New York: Basic Books.

Gimber, Arno y LóPez-VArela, Asunción. "Multimodality, Intermediality, Theory, Practice and Performance" Comparative Review Article. CLCWeb: Comparative Literature and Culture. Purdue University Press Volume 12.3, 2010.

$<$ http://docs.lib.purdue.edu/clcweb/>

Goleman, Daniel. (1995): Emotional intelligence. New York: Bantam Books.

Gross, James (2006): Handbook of Emotion Regulation. The Guilford Press.

Hayles, N. Katherine (2002): Writing Machines. Cambridge: MIT Press.

Heidegger, Martin (2005 / 1962): Being and Time. Trans. Joan Stambaugh. Blackwell Publishing.

Hedling, Erik \& Lagerroth, Ulla Britta (eds) (2002): Cultural Functions of Intermedial Exploration. Amsterdam: Rodopi. 
Helbig, Jörg (ed.) (1998): Intermedialität: Theorie und Praxis eines interdisziplinären Forschungsgebiets. Berlin: Schmidt Also online: <http://www.uni-koeln.de/philfak/englisch/helbig/inhalt.htm>

HigGS, Malcolm \& Hender, Jill (2004): "The Characteristics of the Creative Manager." Journal of General Management, Volume 29, Number 4, págs. 1-20.

Higgins, Dick. (1984): Horizons. The Poetics and Theory of the Intermedia. Carbondale and Edwardsville: Southern Illinois University Press.

Husserl, Edmund (1970): The Crisis of European Sciences and Transcendental Phenomenology. Trans. D. Carr. Evanston: Northwestern University Press.

HutTo, Daniel (2009): "Folk Psychology as Narrative Practice." Journal of Consciousness Studies, 16, No. 6-8, págs. 9-39

KANT, Immanuel (1961 / 1781): "The critique of pure reason". En W. Kaufmann (Ed.) Philosophic classics: Bacon to Kant. New Jersey: Prentice-Hall.

KEMMERER, David (2006): "The semantics of space: integrating linguistic typology and cognitive neuroscience”. Neuropsychology, 44 págs. 1607-1621.

KnuUtTila, Simo (2004): Emotions in Ancient and Medieval Philosophy. USA: Oxford University Press.

Kunn, Thomas (1970): The Structure of Scientific Revolutions. $2^{\text {nd }}$. Chicago. University of Chicago Press.

LACAN, Jacques, (1982 / 1953): “Le Symbolique, l'Imaginaire et le Réel”. Bulletin de l'Association freudienne, . $^{\circ} 1$, págs. 4-13.

,.(1991 / 1960-61) Le Séminaire. Livre VIII. Le transfert, (Ed.) Jacques-Alain Miller. Paris: Seuil.

Landow, George (2005): Hypertext 3.0: Critical Theory and New Media in the Era of Globalization. Johns Hopkins University Press.

(2009): "Real Cities, Virtual Cities: Model Railroading as Analogue Virtual Reality." In López-Varela, Asunción \& Mariana Net (Eds.) Real and Virtual Cities: Intertextual and Intermedial Mindscapes. Bucharest: Univers Enciclopedic, págs. 35-67

LÓPEZ-VARELA, Asunción (2004): Embers of Time: a pluridisciplinary exploration of the crisis of representacional time in science and literature. Doctoral dissertation. Universidad Complutense.

, (Ed.) (2008): Cultural Scenarios of the Fantastic. Monographic issue. CLCWeb: Comparative Literature and Culture 10.4 Purdue University Press $<$ http://docs.lib.purdue.edu/clcweb/vol10/>

(2009): "E-motions and the Pleasures of the Interface: The Rapprochement of Technology, Art and Education". En Verena Laschinger and Ralph J. Poole E-motion: Sentiment and Technology. Fatih Univiersity Press, págs. 139-161

- (2010): "Complejidad, tecnología y escenarios culturales de lo fantástico." Pilar Andrade, Arno Gimber y Maria Goicoechea (Eds.) Espacios y Tiempos de lo Fantástico: una mirada al siglo XXI. Bern: Peter Lang. págs. 179-191

(2011a) "Intermedial Cognitive Semiotics": En Material Aesthetics, Media Formats and Cultural Studies. Tötösy, S.; López-Varela, A.; Sassy, H.; Miszkowski, J. (eds.) Monographic issue. CLCWeb: Comparative Literature and Culture 13.3 Purdue University Press <http://docs.lib.purdue.edu/clcweb/vol13/3>

- (2011b) "Multimodal Metaphor and Intersubjective Experiences: the importance of eye-contact in Davison's graphic novel The Spiral Cage and in Annie Abrahams net-project On Collaboration. En Masucci, Lello e Di Rosario, Giovanna, Lavori del convegno Palazzo degli Artista italiani. Oficina di Letterature Electrónica. Napoli, págs 307-324. 
LóPEZ-VArela, Asunción y Net, Mariana (Eds.) (2009): Real and Virtual Cities: Intertextual and Intermedial Mindscapes. Bucharest: Univers Enciclopedic.

LÓPEZ-VARELA, Asunción y TÖTÖSY, Steven (2009): "Real and Imagined Urban Spaces and Transformations in/of Western Society" en Mariana Net Urban Spaces and Transformations in/of Western Society TRANS. 16 Internet-Zeitschrift für Kulturwissenschaften /Internet Journal of Cultural Studies Journal of the Research Institute for Austrian and International Literature and Cultural Studies (INST)

- (2010): “Comparative Cultural Studies, éducation, nouveaux médias et l'interculturalisme." Anne Chalard-Fillaudeau (Ed. \& Trans). Le Complexe français. Quelle place pour les études et sciences de la culture en France? Ed. Anne Chalard-Fillaudeau., Revue d'Etudes Culturelles, Dijon, Abell, n. 5, págs. 73-93.

Manovich, Lev (2001): The Language of the Media. Cambridge, Mass. and London: MIT

MAYER, Richard E (2005): The Cambridge handbook of multimedia learning. Cambridge University Press.

McLluhan, Marshall (1964): Understanding media: the extensions of man. London: Routledge.

MEAD, George. (1967 /1934): H. Mind, self, \& society. (Ed.) Charles W. Morris. University of Chicago Press.

Merleau-Ponty, Maurice. (1945): La Phénoménologie de la perception, Paris, Gallimard.

Morganti, Francesca, Carassa, Antonella y Riva, Giuseppe (Eds.) (2008): Enacting Intersubjectivity: A Cognitive and Social Perspective on the Study of Interactions. IOS Press, <http://www.neurovr.org/emerging/volume10.html>

MüLLER, Jürgen E. (1996): Intermedialität. Formen moderner kultureller Kommunikation. Münster: Nodus.

- (2002): "Mediengeschichte intermedial: Perspektiven, Postulate, Prognosen," in Frank Furtwängler et al. (eds.), Zwischen-Bilanz. Eine Festschrift zum 60. Geburtstag von Joachim Paech, <http://www.uni-konstanz.de/paech2002>

Murray, Janet H. (2003): "Inventing the Medium" The New Media Reader, Eds. NoahWardrip-Fruin and Nick Montfort. MIT, págs. 3-5.

OAtLey, Keith y Jenkins, Jennifer. M. (1996): Understanding Emotions, Oxford: Blackwell.

ONG, Walter J. (1982): Orality and literacy: the technologizing of the Word. London: Routledge.

OOSSTERLING, Henk (2003): "Sen(a)ble Intermediality and Interesse. Towards an Ontology of the In-Between." Intermedialités, no. 1, págs. 29-46.

$<$ http://cri.histart.umontreal.ca/cri/fr/intermedalities/Oosterling.pdf $>$

OvergaArd, Søren (2007): Wittgenstein and Other Minds: Rethinking Subjectivity and Intersubjectivity with Wittgenstein, Levinas, and Husserl, London: Routledge.

PAECH, Joachim (1997): "Paradoxien der Auflösung und Intermedialität." HyperKult. Geschichte, Theorie und Kontext digitaler Medien, (ed.) Martin Warnke, Wolfgang Coy und Georg Christoph Tholen. Basel \& Frankfurt a.M.: Stroemfeld/Nexus, págs. 331-368.

Plett, Heinrich F. (1991): “Intertextualities.” En Heinrich F. Plett (ed.), Intertextuality, Berlin, New York. De Gruyter, págs. 3-29.

PRÜMM, Karl (1988): "Intermedialität und Multimedialität." En Ansichten einer künftigen Medienwissenschaft, (ed.) Volker Bohn \& Eggo Müller \& Hans Ruppert. Berlin, págs. 195-200.

PunZI, Maddalena P. (2007): Literary Intermediality. Germany: Peter Lang. 
Pylyshyn, Zenon W. (2000): "Situating vision in the World." Trends in Cognitive Sciences 4.5, págs. 107-207.

RAJEWSKY, Irina (2002): Intermedialität. Tübingen: Francke.

RENSINK, Ronald (2000): “The dynamic representation of scenes.” Visual Cognition 7, págs. $17-42$.

Rodríguez-VIRgiLi, Jordi, Sádaba, Teresa y López-Hermida, Alberto (2010): "La ficción audiovisual como nuevo escenario para la Comunicación Política". Cuadernos de Información y Comunicación vol. 15, págs. 37-54.

Ricoeur, Paul (1969): Le conflit des interprétations. Essais d'herméneutique I, París.Le Seuil.

SARTRE, Jean Paul (1956 / 1943) Being and Nothingness: A Study in Phenomenological Ontology. Trans. Hazel Barnes. NY: New York Philosophical Library.

SCHRÖTER, Jens (2008): "Das ur-intermediale Netzwerk und die (Neu-)Erfindung des Mediums im (digitalen) Modernismus." En Intermedialität analog/digital. Theorien Methoden - Analysen, (ed.) Joachim Paech/Jens Schröter, München: Fink, págs. 579-601.

- (2010): "The Politics of Intermediality." En Film and Media Studies. Scientific Journal of Sapientia University 2, págs. 107-124.

- (2011): "Discourses of Intermediality". En Material Aesthetics, Media Formats and Cultural Studies. (eds.) Tötösy, S.; López-Varela, A.; Sassy, H.; Miszkowski, J. Monographic issue. CLCWeb: Comparative Literature and Culture 13.3 Purdue University Press <http://docs.lib.purdue.edu/clcweb/vol13/3>

SEARLE, John R. (1995): The Construction of Social Reality. London: Allen Lane.

SpIElmanN, Yvonne (1998): "Intermedia and the Organization of the Image: Some Reflections on Film, Electronic, and Digital Media," Iris, nº. 25, spring, págs. 61-74.

SternberG, Meir (2003): "Universals of Narrative and Their Cognitivist Fortunes (I)" Poetics Today Volume 24, Number 2, págs. 297-395.

WeISINGER, Hendrie, D. (1998): Emotional Intelligence at Work. San Francisco: Jossey-Bass. Weisstein, Ulrich (1993): "Literature and the (Visual) Arts. Intertextuality and Mutual Illumination," Ingeborg Hoesterey and Ulrich Weisstein (eds.), Intertextuality: German Literature and Visual Art from Renaissance to the Twentieth Century, Columbia, South Carolina, Camden House.

WERTSCH, James V. (1991): Voices of the Mind: A sociocultural approach to mediated action. Cambridge, MA: Harvard University Press.

Wolf, Werner. (2002): "Towards a Functional Analysis of Intermediality: The Case of Twentieth-Century Musicalized Fiction”. Cultural Functions of Intermedial Exploration. Ed. Erik Hedling and Ulla Britta Lagerroth. Amsterdam: Rodopi, págs. 15-34.

YALKUT, Jud (1973): “Understanding Intermedia." En Avantgardistischer Film 1951-1971. Theorie, ed. Gottfried Schlemmer. München: Hanser, págs. 92-95.

ZlateV, Jordan; Racine, Timothy; Sinha, Chris and Itkonen, Esa (Eds.) (2008): The Shared Mind. Perspectives in Intersubjectivity. Amsterdam, Philadelphia: John Benjamins Publishing Company. 


\title{
RESUMEN
}

En el contexto contemporáneo en el que nos encontramos inmersos en una movilidad continua, tanto física como ideológica, mediada por la velocidad de los medios de transporte de personas y de datos (Internet) a través no solo de las fronteras físicas de nuestro cuerpo, sino de las fronteras territoriales de nuestras comunidades, el impacto de la investigación en intersubjetividad se encuentra necesariamente vinculado a los cambios en los medios materiales que propician los intercambios comunicativos, es decir, a la noción de intermedialidad. Este artículo busca presentar un esbozo del complejo entramado que vincula ambos conceptos.

Palabras clave: Cognición, constructivismo, estudios culturales comparados, intermedialidad, intersubjetividad, interculturalidad, semiótica.

\begin{abstract}
In the contemporary context in which we are immersed in a seamless mobility, both physical and ideological, mediated by the speed of transportation of people and data (Internet) through the physical boundaries of our body and the territorial borders of our communities, the impact of research on intersubjectivity is necessarily linked to changes in media materials that promote communicative exchanges, that is, to the notion of intermediality. This article seeks to present an outline of the complex network that relates both concepts.
\end{abstract}

Key words: cognition, constructivism, compartive cultural studies, intermediality, intersubjectivity, interculturalism, semiotics.

\section{RÉSUMÉ}

Dans le contexte actuel dans lequel nous sommes immergés dans une mobilité sans couture, à la fois physique et idéologique, médiée par la vitesse de transport des personnes et des données (Internet), grâce à des limites physiques de notre corps et des frontières territoriales de nos communautés, l'impact de la recherche sur l'intersubjectivité est nécessairement liée à l'évolution des matières médiatiques que favorisent les échanges de communication, c'est-à-dire, à la notion de intermédialité. Cet article vise à présenter les grandes lignes du réseau complexe qui concerne les deux concepts.

Mots clé: Cognition, constructivisme, comparative études culturelles, intermédialité, intersubjectivité, interculturalité, sémiotique. 